\title{
Correlated tunneling in intramolecular carbon nanotube quantum dots
}

\author{
M. Thorwart, ${ }^{1}$ M. Grifoni, ${ }^{1}$ G. Cuniberti, ${ }^{2}$ H. W. Ch. Postma,,${ }^{1}$ 用 and C. Dekker ${ }^{1}$ \\ ${ }^{1}$ Department of NanoScience, Delft University of Technology, Lorentzweg 1, 2628 CJ Delft, The Netherlands \\ ${ }^{2}$ Max Planck Institute for the Physics of Complex Systems, D-01187, Dresden, Germany
}

(Dated: November 6, 2018)

\begin{abstract}
We investigate correlated electronic transport in single-walled carbon nanotubes with two intramolecular tunneling barriers. We suggest that below a characteristic temperature the long range nature of the Coulomb interaction becomes crucial to determine the temperature dependence of the maximum $G_{\max }$ of the conductance peak. Correlated sequential tunneling dominates transport yielding the power-law $G_{\max } \propto T^{\alpha_{\text {end-end }}-1}$, typical for tunneling between the ends of two Luttinger liquids. Our predictions are in agreement with recent measurements.
\end{abstract}

PACS numbers: 71.10.Pm, 71.20.Tx, 72.80.Rj

Electronic correlations have been predicted to dominate the characteristic features in single-walled metallic carbon nanotubes (SWNTs) [1, 2, which has recently been observed in experiments [3, 4, 5]. The onedimensional nature of the electronic conduction bands reveals itself in typical Luttinger liquid [6], rather than Fermi liquid behavior. The manipulation of individual nanotubes with an atomic force microscope permits the creation of intra-tube buckles acting as tunneling barriers [5]. Recently, SWNTs with two intramolecular buckles have been reported to behave as room-temperature single-electron transistors [7]. At low temperatures, the thermal electronic energies are smaller than the level separation between the discrete energy states of the island and tunneling occurs via these discrete levels. Resonant tunneling in Luttinger liquids has been investigated theoretically by many authors $18,9,10,11,12]$. The onedimensional nature of the correlated electrons is responsible for the differences to the quantum Coulomb blockade theory for conventional, e.g., semiconducting quantum dots 13. Varying the gate voltage results in a sequence of conductance peaks. In the (uncorrelated) sequential tunneling (UST) approximation the temperature dependence of the maxima of those peaks follows the power-law [11]

$$
G_{\max } \propto T^{\alpha_{\mathrm{end}}-1}
$$

with $\alpha_{\text {end }}$ being the density of states exponent for tunneling into the end of a Luttinger liquid. However, recent experiments [7] suggest a different power-law,

$$
G_{\max } \propto T^{\alpha_{\text {end-end }}-1}
$$

with $\alpha_{\text {end-end }}=2 \alpha_{\text {end }}$.

In this Letter, we propose that a novel tunneling mechanism, correlated sequential tunneling (CST), gives rise to the power law (2). It originates from the finite range nature of the Coulomb interaction in SWNTs and replaces conventional uncorrelated sequential tunneling. It dominates resonant transport at low temperatures and strong interactions. CST leads to a renormalization of the intrinsic linewidth of the resonance, which is reflected in an increase of $G_{\max }$ with increasing temperature. In contrast, UST would predict the opposite behavior 14. A good agreement with the experimental results $[7]$ is found.

We describe an individual metallic SWNT with two buckles by the Hamiltonian $H=H_{0}+H_{\mathrm{B}}+H_{\text {ext }}$. Here, $H_{0}$ characterizes the one-dimensional homogeneous wire including the finite-range electronic interaction. Metallic carbon nanotubes possess two gap-less one-dimensional bands [1, 2] with Fermi velocity $v_{\mathrm{F}} \simeq 8 \times 10^{5} \mathrm{~m} / \mathrm{s}$ [7, which dominate the low-energy physics. In the bosonized representation, one finds [2]

$$
\begin{aligned}
H_{0} & =\sum_{a=\rho, \sigma, \Delta \sigma, \Delta \rho} \int d x \frac{\hbar v_{\mathrm{F}}}{2}\left[\left(\partial_{x} \vartheta_{a}\right)^{2}+\left(\partial_{x} \phi_{a}\right)^{2}\right] \\
& +\frac{1}{\pi} \int d x \int d x^{\prime} \partial_{x} \vartheta_{\rho}(x) V\left(x-x^{\prime}\right) \partial_{x^{\prime}} \vartheta_{\rho}\left(x^{\prime}\right),
\end{aligned}
$$

containing three sectors of neutral excitations ( $a=$ $\sigma, \Delta \rho, \Delta \sigma)$ propagating with $v_{\mathrm{F}}$, and one sector of charged excitations $(a=\rho)$. The charge sector is characterized by the nonlinear dispersion relation $\omega(k)=$ $v_{\mathrm{F}}|k|\left[1+\hat{V}(k) / \pi \hbar v_{\mathrm{F}}\right]$, where $\hat{V}(k)$ is the Fourier transform of the screened Coulomb potential projected onto the $x$ direction $V(x)$ 17. In our approach, the nonlinear dispersion is crucial, since it modifies the low energy properties of the dynamics. The two tunneling barriers at $x= \pm x_{0} / 2$ are modeled by a scattering potential $U(x)=U_{\mathrm{B}} \sum_{\kappa=\mp} \delta\left(x+\kappa x_{0} / 2\right)$ leading to $H_{\mathrm{B}}=\int d x U(x) \rho(x)$. Here, $\rho(x)$ is the total electron density and backscattering has to be included. Upon introducing $N_{a}=\frac{2}{\sqrt{\pi}}\left[\vartheta_{a}\left(\frac{x_{0}}{2}\right)+\vartheta_{a}\left(-\frac{x_{0}}{2}\right)\right]$ and $n_{a}=\frac{2}{\sqrt{\pi}}\left[\vartheta_{a}\left(\frac{x_{0}}{2}\right)-\vartheta_{a}\left(-\frac{x_{0}}{2}\right)\right]+\frac{4 k_{F} x_{0}}{\pi} \delta_{a, \rho}, H_{\mathrm{B}}$ provides an eight-dimensional sinusoidal potential for the tunneling dynamics associated with the variables $N_{a}$ and $n_{a}$ [8]. Physically, $e N_{\rho} / 2$ counts the unit charges transferred through the dot, while $-e n_{\rho}$ is the total charge accumulated on the dot. Likewise, $N_{\sigma}$ and $n_{\sigma}$ are related to the difference of spin fluctuations between both leads, and to the deviation from the mean value in 
the dot, respectively. The analogous interpretation for $N_{\Delta \sigma}, n_{\Delta \sigma}$ and $N_{\Delta \rho}, n_{\Delta \rho}$ holds. Finally, the gate voltage $V_{\mathrm{G}}$ and the bias voltage $V$ give rise to the term $H_{\mathrm{ext}}=-e\left[(V / 2) N_{\rho}+\lambda V_{\mathrm{G}} n_{\rho}\right]$, where $\lambda=C_{\mathrm{G}} / C_{\mathrm{tot}}$, with $C_{\mathrm{G}}$ the gate capacitance and $C_{\mathrm{tot}}$ the total capacitance of gate and island.

The relevant observable is the asymptotic tunneling current $I=\lim _{t \rightarrow \infty} \frac{e}{2}\left\langle\dot{N}_{\rho}(t)\right\rangle$, where $\langle\ldots\rangle$ denotes the expectation value determined via the reduced density matrix (RDM). The latter is obtained by tracing out exactly the Luttinger modes away from the barriers [18]. The diagonal elements of the RDM are given by the conditional probabilities $P\left(\mathbf{N}_{\mathrm{f}}, t ; \mathbf{N}_{\mathrm{i}}, t_{0}\right)$ of being in the final state $\mathbf{N}_{\mathrm{f}}:=\left(\left\{N_{a, \mathrm{f}}\right\},\left\{n_{a, \mathrm{f}}\right\}\right)$ at time $t$, having started from the state $\mathbf{N}_{\mathrm{i}}$ at time $t_{0}$. Then, we find

$$
I=\frac{e}{2} \lim _{t \rightarrow \infty} \sum_{\mathbf{N}_{\mathrm{f}}} N_{\rho, \mathrm{f}} \dot{P}\left(\mathbf{N}_{\mathrm{f}}, t ; \mathbf{N}_{\mathrm{i}}, t_{0}\right):=e\left(\Gamma^{\mathrm{f}}-\Gamma^{\mathrm{b}}\right),
$$

with $\Gamma^{\mathrm{f} / \mathrm{b}}$ being the total rates for forward/backward transfer of charge. In turn, after exploiting the detailed balance relation $\Gamma^{\mathrm{b}}(V)=e^{-\beta e V} \Gamma^{\mathrm{f}}(V)$, the linear conductance follows as $G=\lim _{V \rightarrow 0} e^{2} \beta \Gamma^{\mathrm{f}}$. Here, $\beta=1 /\left(k_{\mathrm{B}} T\right)$ is the inverse thermal energy.

In general, the conditional probability $P\left(\mathbf{N}_{\mathrm{f}}, t ; \mathbf{N}_{\mathrm{i}}, t_{0}\right)$ can be evaluated exactly in terms of a multiple real time path integral involving forward $N_{a}(\tau)\left(n_{a}(\tau)\right)$ and backward $N_{a}^{\prime}\left(\tau^{\prime}\right)\left(n_{a}^{\prime}\left(\tau^{\prime}\right)\right)$ paths 10. The effect of the bath modes $\vartheta_{a}\left(x \neq \pm x_{0} / 2, \tau\right)$ enters via the influence functionals $\mathcal{F}_{+}=\exp \left\{\sum_{a} \Phi_{+}\left[N_{a}, N_{a}^{\prime}\right]\right\}$, and $\mathcal{F}_{-}=$ $\exp \left\{\sum_{a} \Phi_{-}\left[n_{a}, n_{a}^{\prime}\right]+\Phi_{\mathrm{C}}\left[n_{a}, n_{a}^{\prime}\right]\right\}$, where the phases

$$
\begin{aligned}
\Phi_{ \pm}\left[q, q^{\prime}\right]= & \int_{t_{0}}^{t} d \tau \int_{t_{0}}^{t} d \tau^{\prime}\left[\dot{q}(\tau)-\dot{q}^{\prime}\left(\tau^{\prime}\right)\right] \\
& \times\left\{W_{ \pm}^{a}\left(\tau-\tau^{\prime}\right) \dot{q}\left(\tau^{\prime}\right)-W_{ \pm}^{a *}\left(\tau-\tau^{\prime}\right) \dot{q}^{\prime}\left(\tau^{\prime}\right)\right\}(5)
\end{aligned}
$$

induce nonlocal-in-time correlations among different tunneling transitions. The correlation functions read [18]

$W_{ \pm}^{a}(t)=\int_{0}^{\infty} d \omega \frac{J_{ \pm}^{a}(\omega)}{\omega^{2}}\left[(1-\cos \omega t) \operatorname{coth} \frac{\hbar \beta \omega}{2}+i \sin \omega t\right]$,

with the spectral densities [10] $J_{ \pm}^{a}(\omega)=\omega e^{-\omega / \omega_{c}}[1 / 2+$ $\left.\left(\Delta E_{\sigma} / \hbar\right) \sum_{m=1}^{\infty} \delta\left(\omega-\Omega_{ \pm}(m)\right)\right] / 4$ for the neutral sectors $(a=\sigma, \Delta \sigma, \Delta \rho)$. Here, we have introduced a cut-off frequency $\omega_{c}$, and $\Omega_{+}(m)=\Delta E_{\sigma}(2 m-1) / \hbar, \Omega_{-}=$ $\Delta E_{\sigma} 2 m / \hbar$. Finally, $\Delta E_{\sigma}=\pi \hbar v_{\mathrm{F}} / x_{0}$ is related to the energy quantization of the neutral plasmon modes in the dot. In [7], $\Delta E_{\sigma}>k_{\mathrm{B}} T$ also at room temperature. For the charged sector the spectral density reads $J_{ \pm}^{\rho}(\omega)=-K^{ \pm}\left(\omega_{n} \rightarrow-i \omega+0^{+}\right) / \pi \hbar$, where

$$
\left[K^{ \pm}\left(\omega_{n}\right)\right]^{-1}=\frac{4 v_{\mathrm{F}}}{\hbar \pi} \int_{0}^{\infty} d k \frac{1 \pm \cos \left(k x_{0}\right)}{\omega_{n}^{2}+\omega^{2}(k)},
$$

and $\omega_{n}$ are the Matsubara frequencies. For an arbitrary finite range potential the spectral densities $J_{ \pm}^{\rho}(\omega)$ cannot be evaluated analytically. In general, as in the zero-range case, $V_{\mathrm{zr}}(x)=V_{0} \delta(x)$, the leads contribute an Ohmic spectrum at low frequency: $\lim _{\omega \rightarrow 0} J_{+}^{\rho}(\omega)+J_{-}^{\rho}(\omega):=$ $J_{\Sigma}^{\rho}(\omega)=\omega / g_{\rho}$, where $g_{\rho}^{-2}=1+\hat{V}(k=0) / \pi \hbar v_{\mathrm{F}}$. Theoretical estimates as well as experiments show that typical values for $g_{\rho}$ range between 0.18 and 0.26 in SWNTs 过, 2, 急, 田, being well below $g_{\rho}=1$ for noninteracting electrons.

As a consequence of the finite range interaction, a new energy scale arises which introduces two qualitatively new features: (i) The $\delta$-shaped resonances for the zerorange limit turn into resonances of finite height and with an intrinsic line-width $\propto 1 / g_{\Delta}$, cf. Fig. 1. (ii) The new parameter $g_{\Delta}^{-1}$ is defined from the low energy relation

$$
\lim _{\omega \rightarrow 0} J_{+}^{\rho}(\omega)-J_{-}^{\rho}(\omega):=J_{\Delta}^{\rho}(\omega)=\omega / g_{\Delta} .
$$

For ease of calculation, we assume a potential $V(x)=$ $V_{0} a / 2 \exp (-a|x|)$ [17, with $a$ being the inverse screening length $(a \rightarrow \infty$ yields the zero-range interaction limit $V_{0} \delta(x)$ and implies $g_{\Delta}^{-1} \rightarrow 0$ ). The numerical results for this potential are shown in Fig. 1. Note however that, as shown below, our main conclusions are independent of the precise shape of the potential. Finally, $\Phi_{\mathrm{C}}$ provides a quadratic local-in-time contribution proportional to the addition energies $E_{\sigma}=\Delta E_{\sigma} / 2$ for the neutral sectors, and $E_{\rho}=K^{-}\left(\omega_{n}=0\right)$ for the charge plasmons.

Of foremost importance is how to evaluate the forward rate $\Gamma^{\mathrm{f}}$. In the large barrier limit, the dynamics is ruled by well separated tunneling events between the adjacent minima of the periodic potential in $H_{\mathrm{B}}$. Each tunneling event induces the change $N_{a} \rightarrow N_{a} \pm 1$, and $n_{a} \rightarrow n_{a} \pm 1$. In particular, $N_{\rho} \rightarrow N_{\rho} \pm 1$ describes one discrete charge tunneling through one barrier from left to right (-) or vice versa $(+)$, while $n_{\rho} \rightarrow n_{\rho} \pm 1$ describes a unit charge tunneling onto/out of the dot. Each tunneling event through the left (right) barrier contributes a factor $i \Delta_{\mathrm{L}}\left(-i \Delta_{\mathrm{R}}\right)$, where $\Delta_{\mathrm{L}}=\Delta_{\mathrm{R}}$ is the associated tunneling amplitude. To proceed, we express the double path integrals over the paths $N_{a}, N_{a}^{\prime}$ and $n_{a}, n_{a}^{\prime}$ as single path integrals over paths along the states of the $\mathrm{RDM}$ in the $\left(N_{a}, N_{a}^{\prime}\right)$ - and in the $\left(n_{a}, n_{a}^{\prime}\right)$-planes. According to the boundary conditions, all paths start and end in diagonal states of the RDM, making intermittent visits to off-diagonal states, cf. Fig. 2. Due to the strong Coulomb interaction $\left(g_{\rho}<1\right)$, the relevant paths are those for which the system is back to a diagonal state after two tunneling events [18]. We focus on the linear regime $(V \rightarrow 0)$, so that either $n_{\rho}=n$ or $n_{\rho}=n+1$ units of charge are allowed in the dot in the stationary state [8]. The current (4) is then expressed as a series expansion in the tunneling amplitudes $\Delta_{\mathrm{R} / \mathrm{L}}$. To lowest order, the forward rate is divergent. The divergent term 
reads

$$
\begin{aligned}
& \Gamma^{\mathrm{f},(4)}=\lim _{\lambda \rightarrow 0} \frac{\Delta_{\mathrm{L}}^{2} \Delta_{\mathrm{R}}^{2}}{8} \int_{0}^{\infty}\left(\prod_{i=1}^{3} d \tau_{i}\right) e^{-\lambda \tau_{2}-S_{\Sigma}\left(\tau_{1}\right)-S_{\Sigma}\left(\tau_{3}\right)} \\
& \times \cosh \Lambda_{13}^{\Delta} \cos \mathcal{R}_{13}^{\Delta}\left[c_{\mathrm{Lf}}\left(\tau_{1}\right) c_{\mathrm{Rf}}\left(\tau_{3}\right)+c_{\mathrm{Rf}}\left(\tau_{1}\right) c_{\mathrm{Lf}}\left(\tau_{3}\right)\right],(9)
\end{aligned}
$$

where $\tau_{i}$ denote the time intervals elapsed between the different tunneling events. Then, $\tau_{2}$ is the time spent in the intermediate diagonal state. We use the notation $c_{\kappa \alpha}(\tau)=\cos \left(R_{\Sigma}(\tau)-E_{\kappa, \alpha} \tau\right)$, where the energies are defined as $E_{\mathrm{L}, \mathrm{f}}(n)=-\mu(n+1)-e V / 2$ and $E_{\mathrm{R}, \mathrm{f}}(n+1)=$ $\mu(n+1)-e V / 2$. Here, the chemical potential reads $\mu(n+$ $1)=E_{\rho}\left(n-n_{0}-C_{\mathrm{G}} V_{\mathrm{G}} / e+1 / 2\right)+(3 / 2) E_{\sigma}\left(\sigma_{n+1}^{2}-\sigma_{n}^{2}\right)$, with $n_{0}=4 k_{\mathrm{F}} x_{0} / \pi$ being the mean electron number on the dot. Moreover, $\sigma_{n}=0( \pm 1)$ for an even (odd) number of spins in the dot. The correlations are encapsulated in $S_{\Sigma / \Delta}(\tau)$ and $R_{\Sigma / \Delta}(\tau)$ being the real and imaginary parts, respectively, of $W_{\Sigma / \Delta} \equiv \sum_{a}\left(W_{+}^{a} \pm W_{-}^{a}\right)$. The long range nature of the Coulomb interaction is reflected in the correlators $\Lambda_{13}^{\Delta}$, and $\mathcal{R}_{13}^{\Delta}$, inducing dipoledipole correlations between the tunneling events across the left and right barrier, cf. Fig. 2. To be definite, $\Lambda_{13}^{\alpha}=$ $S_{\alpha}\left(\tau_{1}+\tau_{2}+\tau_{3}\right)+S_{\alpha}\left(\tau_{2}\right)-S_{\alpha}\left(\tau_{1}+\tau_{2}\right)-S_{\alpha}\left(\tau_{2}+\tau_{3}\right)$, and $\mathcal{R}_{13}^{\alpha}=R_{\alpha}\left(\tau_{1}+\tau_{2}+\tau_{3}\right)-R_{\alpha}\left(\tau_{2}+\tau_{3}\right)+R_{\alpha}\left(\tau_{1}+\tau_{2}\right)-R_{\alpha}\left(\tau_{2}\right)$. Here, $\alpha=\Delta$ if the interaction is between tunneling through different barriers, while $\alpha=\Sigma$ if events through the same barrier are involved. Due to (8), the correlators associated to $W_{\Delta}^{\rho}$ behave as $S_{\Delta}^{\rho}(\tau) \rightarrow \pi k_{\mathrm{B}} T \tau / g_{\Delta} \hbar$, and $R_{\Delta}^{\rho} \rightarrow$ const. at long times. Hence, $\Lambda_{13}^{\Delta, \rho} \rightarrow 0$ and $\mathcal{R}_{13}^{\Delta, \rho} \rightarrow 0$ when $\tau_{2} \rightarrow \infty$. The functions $S_{\Delta}^{a}$ and $R_{\Delta}^{a}$ associated to the neutral sectors are purely periodic with frequency $\Delta E_{\sigma} / \hbar$.

To cure the divergence of the fourth order rate, we have to sum up divergent irreducible terms of higher order. Therefore, appropriate approximation schemes are required. We make the following approximations: (i) We only consider higher order paths which yield the UST result for vanishing interaction range. (ii) We neglect the dipole-dipole correlations $\Lambda_{i j}^{\Sigma}$ due to $g_{\rho} \ll 1$, but keep the correlations $\Lambda_{i j}^{\Delta}$ among the inner dipoles up to linear order. iii) We consider a large cut-off $\omega_{c}$ (scaling limit), and neglect the correlations $\mathcal{R}_{i j}^{\Delta, \rho}$. The sum over the so obtained higher order irreducible paths yields for the total rate $\Gamma^{\mathrm{f}}=\sum_{n=2}^{\infty} \Gamma^{\mathrm{f},(2 n)}$ a finite result. It can be obtained from (9) upon replacing $\Lambda_{13}^{\Delta} \rightarrow 3 \Lambda_{13}^{\Delta, \sigma}, \mathcal{R}_{13}^{\Delta, \rho}=0$, and $\lambda \rightarrow \lambda+\gamma$. Here $\gamma$ can be interpreted as a linewidth, i.e., $\gamma=\Gamma^{\mathrm{tot}}+\gamma^{\Delta}$, where $\Gamma^{\mathrm{tot}}=\Gamma^{\mathrm{Rf}}+\Gamma^{\mathrm{Lf}}+\Gamma^{\mathrm{Rb}}+\Gamma^{\mathrm{Lb}}$, and $\Gamma^{\kappa \alpha}=\left(\Delta_{\kappa}^{2} / 2\right) \int_{0}^{\infty} d \tau e^{-S_{\Sigma}(\tau)} c_{\kappa \alpha}(\tau)$ are the incoherent rates for forward/backward tunneling through the right/left barrier $\left(E_{\mathrm{L}, \mathrm{f}}(n)=-E_{\mathrm{L}, \mathrm{b}}(n+1), E_{\mathrm{R}, \mathrm{b}}(n)=\right.$ $\left.-E_{\mathrm{R}, \mathrm{f}}(n+1)\right)$. In contrast, $\gamma^{\Delta}$ is the lowest order correction to the linewidth due to the finite range interaction.
It reads at resonance $\left(E_{\kappa, \alpha}=0\right)$

$$
\begin{aligned}
\gamma^{\Delta} & =\frac{\Delta_{\mathrm{R}}^{2} \Delta_{\mathrm{L}}^{2}}{4} \int_{0}^{\infty} \prod_{i=1}^{3} d \tau_{i} e^{-S_{\Sigma}\left(\tau_{1}\right)-S_{\Sigma}\left(\tau_{3}\right)} e^{-\Gamma^{\mathrm{tot}} \tau_{2}} \\
& \times \sin R_{\Sigma}\left(\tau_{1}\right) \sin R_{\Sigma}\left(\tau_{3}\right) \Lambda_{13}^{\Delta, \rho} \cosh 3 \Lambda_{13}^{\Delta, \sigma} .
\end{aligned}
$$

The incoherent rates yield at resonance to the powerlaw $\Gamma^{\kappa, \alpha} \propto T^{\alpha_{\text {end }}}$, where $\alpha_{\text {end }}=1 / g_{\text {eff }}-1$, and $1 / g_{\mathrm{eff}}=\left(1 / g_{\rho}+3\right) / 4$. In the limit $\Lambda_{13}^{\Delta, \rho}=0$ one recovers the known UST rate [11, 12] $\Gamma^{\mathrm{f}}=$ $\Gamma^{\mathrm{Rf}} \Gamma^{\mathrm{Lf}} / \Gamma^{\mathrm{tot}} \propto T^{\alpha_{\text {end }}}$, which implies the power law (11) for $G_{\max }$. While $\Gamma^{\text {tot }}$ decreases with decreasing temperature, $\gamma^{\Delta}$ remains constant. Hence, depending on $g_{\text {eff }}$ a cross-over temperature $T^{*}$ can be identified, with $k_{\mathrm{B}} T^{*} / \hbar \omega_{c}=\left(\gamma^{\Delta} / d_{g_{\text {eff }}}\right)^{1-1 / g_{\text {eff }} / 2 \pi}$ with $d_{g_{\text {eff }}}=$ $\Delta^{2}\left|\Gamma\left(1 / 2 g_{\text {eff }}\right)\right|^{2} /\left(\omega_{c} \Gamma\left(1 / g_{\text {eff }}\right)\right)$. For $T<T^{*}$, the powerlaw (2) follows. Due to the nonlinearity of the spectral densities $J_{\Sigma / \Delta}^{\rho}(\omega)$, we are able to compute the triple integral numerically only for rather large inverse screening lengths $a$, since then the correlators $W_{\Sigma / \Delta}^{\rho}(\tau)$ are well fitted by those of a damped harmonic oscillator. The result for $a=200 x_{0}^{-1}$, yielding $g_{\Delta}^{-1}=0.087$, is shown in the right inset of Fig. 1 for $g_{\rho}=0.23$. Turning to the experiment in [5] we should a tyical screening length $a^{-1} \approx 3-5 \mathrm{~nm}$. For a dot length $x_{0}=25 \mathrm{~nm}$ it corresponds to values $a \simeq 10 x_{0}^{-1}$. However, due to the strong asymmetry of the peaks of the spectral densities $J_{\Sigma / \Delta}^{\rho}$ at $a \simeq 10 x_{0}^{-1}$, a numerical evaluation of $\gamma^{\Delta}$ is no longer possible. In the following, we assume $a=200 x_{0}^{-1}$.

We now compare the outcomes of (9) with the experimental results [7]. Induced buckles on a SWNT define an island of $\sim 25 \mathrm{~nm}$ length. We include the contact influence via the Landauer formula, in which the total tube conductance is convoluted with the Fermi distribution from the Au-leads. With typical Luttinger parameters in the range $g_{\rho} \approx 0.18-0.26$, the agreement between the measured data and the theoretical prediction is very good upon choosing $g_{\rho}=0.23$, leading to $g_{\text {eff }}=0.54$. The comparison is depicted in Fig. 3 together with the power law (2). Note that cotunneling events, which should dominate the tails of a conductance peak [9, 10, 19], are not considered here. The absolute value of $G_{\max }$ is fitted by the parameter $\Delta_{\mathrm{L} / \mathrm{R}}$ (we have chosen $\hbar \omega_{\mathrm{c}}=9 \Delta E_{\sigma}$ ). Importantly, we find a very good agreement between theoretical and experimental results for $k_{\mathrm{B}} T \ll \Delta E_{\sigma}$ with the only one relevant free parameter $g_{\rho}$.

In the regime $k_{\mathrm{B}} T \simeq \Delta E_{\sigma}$, we observe a deviation from the power law due to the contribution of excited plasmon levels which become thermally accessible, as well as due to a crossover to the UST limit. The result of our simple model with two $\delta$-shaped tunneling barriers are fitted to the data. We find $x_{0}=71 \mathrm{~nm}\left(\Delta E_{\sigma}=23 \mathrm{meV}\right)$ which agrees with the measured value of $x_{0}=25 \mathrm{~nm}$. A smaller value for $x_{0}$ only shifts the onset of the deviation from the power-law to higher temperatures. Indeed, the 
measured height profile of the buckle region suggests that the real scattering potential is more complicated. However, our central result, namely the algebraic behavior at low temperature, is not affected by the precise shape of the impurity potentials. We also fit the theoretically predicted line-shape of a conductance peak to the measured data as displayed in the left inset of Fig. 3 and find $\lambda=0.25$, which coincides with the experimentally determined value. We measure the width of a calculated conductance peak and find that it increases linearly with increasing temperature, in agreement with the measured data (right inset of Fig. 3). To underpin the algebraic behavior, we also show in Fig. 3 the conductance $G^{*}$ obtained by integrating the linear conductance over the gate voltage. Due to the linear increase of the peak width with temperature, the exponent of the power law is increased by one, in clear agreement with the experiment.

In summary, we have shown that the finite range of Coulomb interaction can play a crucial role in the resonant tunneling in one-dimensional quantum dots.

We thank U. Weiss and Yu. Nazarov for discussions and T. Teepen and Z. Yao for experimental assistance. We acknowledge support by the Dutch FOM, the German DFG-Emmy-Noether Programm (M.T.), the EC program SATURN, and the Schloeßmann Foundation (G.C.).

* Current address: Condensed Matter Physics 114-36, California Institute of Technology, Pasadena, CA 91125

[1] R. Egger and A. O. Gogolin, Phys. Rev. Lett. 79, 5082 (1997); Eur. Phys. J. B 3, 281 (1998).

[2] C. Kane, L. Balents and M. P. A. Fisher, Phys. Rev. Lett. 79, 5086 (1997).

[3] M. Bockrath et al., Nature (London) 397, 598 (1999).

[4] Z. Yao et al., Nature (London) 402, 273 (1999).

[5] H. W. Ch. Postma et al., Phys. Rev. B 62, R10653 (2000).

[6] J. Voit, Rep. Prog. Phys. 57, 977 (1994).

[7] H. W. Ch. Postma et al., Science 293, 76 (2001).

[8] C. L. Kane and M. P. A. Fisher, Phys. Rev. B 46, 15233 (1992); 46, 7268 (1992).

[9] A. Furusaki and N. Nagaosa, Phys. Rev. B 47, 3827 (1993).

[10] M. Sassetti, F. Napoli, and U. Weiss, Phys. Rev. B 52, 11213 (1995).

[11] A. Furusaki, Phys. Rev. B 57, 7141 (1998).

[12] A. Braggio et al., Europhys. Lett. 50, 236 (2000)

[13] C. W. J. Beenakker, Phys. Rev. B 44, 1646 (1991).

[14] Recently, Kleimann et al. [15] ascribed the unexpected power-law (2) to tunneling between the ends of the Auleads and the SWNT, assuming high contact resistances. However, we measure [7] low contact resistances of $\sim 25$ $\mathrm{k} \Omega$ at $300 \mathrm{~K}$. Moreover, for the SWNT with Au-contacts, we typically find bulk tunneling [16].

[15] T. Kleimann et al., Phys. Rev. B, to be published; see also cond-mat/0208384.

[16] Z. Yao, C. L. Kane, and C. Dekker, Phys. Rev. Lett. 84,

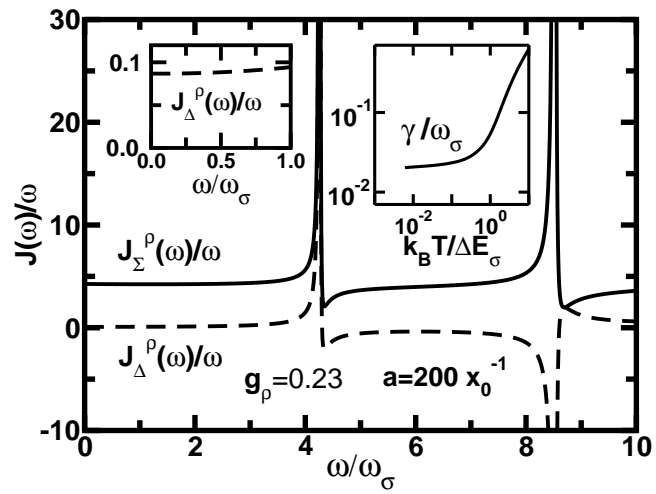

FIG. 1: Spectral densities $J_{\Sigma / \Delta}^{\rho}(\omega) / \omega$ for finite interaction range $\left(a=200 x_{0}^{-1}, g_{\rho}=0.23\right)$ with $\omega_{\sigma} \equiv \Delta E_{\sigma} / \hbar$. Left inset: Enlargement of the low frequency part. The important parameter is $1 / g_{\Delta}=\lim _{\omega \rightarrow 0} J_{\Delta}^{\rho}(\omega) / \omega$. Right inset: Contributions to the effective line-width $\Gamma^{\text {tot }}$ and $\gamma^{\Delta}$, with the latter being originated from the finite range of the interaction (see text).
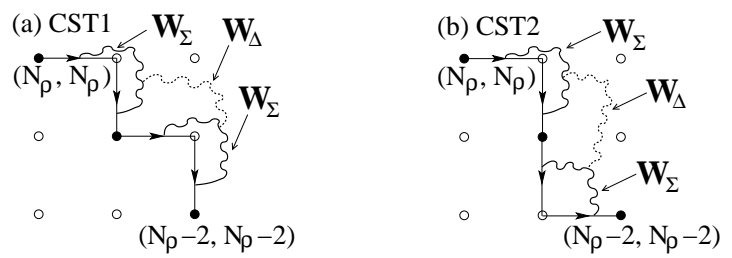

FIG. 2: Lowest order correlated sequential tunneling (CST) paths in the $\left(N_{\rho}, N_{\rho}^{\prime}\right)$-plane for the transfer of one charge through the island corresponding to Eq. (9). Filled circles denote diagonal states of the reduced density matrix.

2941 (2000).

[17] G. Cuniberti, M. Sassetti, and B. Kramer, Phys. Rev. B 57, 1515 (1998).

[18] U. Weiss, Quantum Dissipative Systems (World Scientific, Singapore, 2nd ed., 1999).

[19] D. V. Averin and Yu. V. Nazarov, Phys. Rev. Lett. 65, 2446 (1990). 


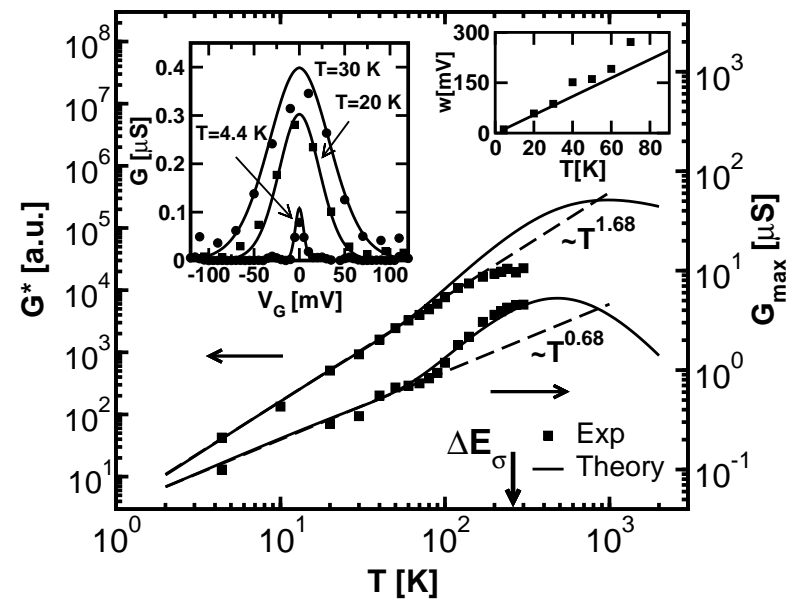

FIG. 3: Conductance maximum $G_{\max }$ (right) and integrated conductance $G^{*}$ (left) as a function of temperature. Solid line: theoretical prediction with $\Delta E_{\sigma}=23 \mathrm{meV}$ and interaction strength $g_{\rho}=0.23$. 무 experimental results [7], dashed lines: power laws, see Eq. (2). Left inset: A single conductance peak for varying gate voltage for $T=4.4 \mathrm{~K}, 20 \mathrm{~K}, 30 \mathrm{~K}$. Right inset: Peak width versus temperature. 